\title{
Pulmonary Embolism in Ischemic Stroke
}

\author{
Prasanna Venkatesan Eswaradass, Sadanand Dey, Dilip Singh, Michael D. Hill
}

\begin{abstract}
Silent pulmonary embolism (PE) may be associated with acute ischemic stroke (AIS). We identified 10 patients from 3,132 unique patients (3,431 CT scans). We retrospectively examined CT angiogram of patients with AIS to determine the frequency of concurrent PE in AIS. The period prevalence of PE was 0.32. Seven patients had concurrent PE, whereas three had PE diagnosed 2 days after their AIS presentation. We suspected paradoxical embolism via patent foramen ovale as the cause of stroke in three patients and thrombophilia in four patients. Seven patients had poor outcome including four deaths. CT angiogram stroke protocol images from aortic arch to vertex allows visualization of upper pulmonary arteries and PE detection in AIS.

RÉSUMÉ: Cas d'embolie pulmonaire liés à des accidents ischémiques cérébraux. Des cas d'embolie pulmonaire (EP) silencieuse peuvent être associés à des accidents ischémiques cérébraux (AIC) aigus. À cet effet, nous avons repéré 10 patients sur un total de 3132 patients, lesquels avaient passé un total de 3431 examens de tomodensitométrie. De façon rétrospective, nous avons passé en revue les examens d'angiographie de patients ayant été victimes d'AIC afin de déterminer la fréquence d'EP concomitantes. La période de prévalence des EP était de 0,32. On a pu observer sept patients victimes d'EP concomitantes alors qu'on a diagnostiqué chez trois d'entre eux des EP survenant deux jours après que leurs AIC se sont manifestés. Nous faisons l'hypothèse qu'une embolie paradoxale, par l'entremise du foramen ovale perméable (FOP), est la cause d'un AIC chez trois patients et de la thrombophilie chez quatre autres. Sept patients ont donné à voir une évolution défavorable de leur état de santé, ce qui inclut quatre décès. Enfin, l'analyse menée à la suite d'examens d'angiographie de la crosse aortique au vertex, le tout en vertu d'un protocole de calibration des images, a permis de visualiser les artères pulmonaires supérieures et de détecter des cas d'EP associés à des AIC.
\end{abstract}

Key words: Pulmonary embolism, Acute ischemic stroke, CT angiogram

doi:10.1017/cjn.2017.288

Can J Neurol Sci. 2018; 45: 343-345

Pulmonary embolism (PE) has an annual incidence of 0.50 0.69 per 1,000 in the general population ${ }^{1}$ and is a preventable cause of death among hospitalized patients. The rate of venous thromboembolism (VTE) (both PE and deep venous thrombosis [DVT]) among hospitalized patients with acute ischemic stroke (AIS) between 1979 and 2003 in a hospital discharge survey was found to be $0.51 \%, 0.74 \%$ and $1.17 \%$, respectively. ${ }^{2}$ The mortality related to PE can be as high as $24 \%^{3}$ but early treatment is highly effective.

Deaths occurring in first week after a large stroke are primarily due to direct effect of brain injury, while deaths occurring subsequently are due to preventable medical conditions like PE. ${ }^{4}$ Patients with stroke are at higher risk of VTE due to bed rest, limb paralysis and increased pro-thrombotic state. ${ }^{5}$ Modern preventive therapy for VTE on a stroke unit can substantively reduce the occurrence of this common stroke-related morbidity. ${ }^{6,7}$

Pulmonary embolism may be associated with AIS both as a concurrent or as a consequence of hemiplegia. The etiology of ischemic stroke is undetermined even after extensive investigations in roughly $25 \%$ of patients. These strokes are presumed to be embolic in nature and recently classified as Embolic Strokes of Undetermined Source (ESUS). Paradoxical embolism is believed to be an important cause for ESUS. ${ }^{8}$ Patients with paradoxical embolism are also at an increased risk for PE. Pulmonary VTE can result in increased right heart pressure leading to paradoxical embolism via right-to-left cardiac shunt such as a patent foramen ovale.

We report the modern prevalence of $\mathrm{PE}$ among stroke patients both as a concurrent diagnosis and as a consequence of hemiplegia.

All stroke patients in Calgary receive a stroke protocol CT and multiphase CT angiogram (CTA) (arch to vertex) at presentation.
We retrospectively examined CTA of patients with AIS to determine the frequency of concurrent PE in AIS. A standard CTA stroke protocol examines the neurovasculature from the aortic arch to the vertex of the head, providing additional information on the angiography of the upper lobes of the lung including the hilum. This study was approved by the Conjoint Health Research Ethics Board at the University of Calgary. We screened all CTA studies of patients who presented to Foothills Medical Centre, Calgary with transient ischemic attacks or stroke. To identify the patients, we conducted a text search of our hospital radiology database from January 2015 to May 2016 for the word "pulmonary emboli" or "pulmonary thrombus" or "pulmonary artery thrombus" or "pulmonary thromboembolus" or "pulmonary artery thromboembolus" or "lung infarction" or "pulmonary infarction" in stroke CTA reports. We identified 18 patients with our text search. The CTA images were reviewed by two stroke fellows independently. Eight patients were excluded from study as they had pulmonary artery enlargement without PE. Finally, we identified 10 patients from 3,132 unique patients $(3,431$ scans)

From the Calgary Stroke Program, Department of Clinical Neurosciences, Cumming School of Medicine, University of Calgary, Calgary, AB, Canada (PVE, SD, DS, MDH); Department of Radiology, Cumming School of Medicine, University of Calgary, Calgary, $\mathrm{AB}$, Canada (MDH); Department of Community Health Sciences, Cumming School of Medicine, University of Calgary, Calgary, AB, Canada (MDH); Department of Medicine, Cumming School of Medicine, University of Calgary, Calgary, AB, Canada (MDH); Hotchkiss Brain Institute, Calgary, AB, Canada (MDH).

Received April 17, 2017. Final Revisions Submitted August 13, 2017. Date of ACCEPTANCE OCTOBER 25, 2017.

Correspondence to: Michael D. Hill, Calgary Stroke Program, Department of Clinical Neurosciences, Hotchkiss Brain Institute, University of Calgary, 3330 Hospital Drive NW, Room 2939, Calgary, AB, Canada T2N 4N1. Email: michael.hill@ucalgary.ca 
and reviewed their charts for history and all diagnostic lab tests. Epidemiological profile, clinical presentation, vascular risk factors, imaging findings, treatment details, outcome and laboratory results were noted. Among investigations, transthoracic echocardiogram with bubble study results were specifically examined for the presence of patent foramen ovale. Admitted stroke patients were managed on the stroke unit at Foothills Hospital where it is part of our standard order set to provide pharmacological DVT prophylaxis with once daily subcutaneous enoxaparin or to use intermittent pneumatic compressions stockings where enoxaparin is contraindicated.

Ten unique cases were identified yielding a period prevalence of $\mathrm{PE}$ at 0.32 . The mean age was 61.1 (range 25-88) and men to women ratio was 1 . Stroke severity varied from National Institutes of Health Stroke Scale 4 to 24. Seven patients had concurrent upper lobe or saddle PE diagnosed at AIS presentation and three had PE diagnosed 2 days after hospital admission. DVT was identified in association with PE and AIS in seven patients. Three patients had a combination of femoral vein and popliteal vein DVT, two had femoral vein DVT only and two had popliteal vein DVT only (Table 1).

Admission CTA head and neck stroke protocol detected PE in seven patients, whereas CT chest detected PE in the remaining three patients. Five patients had unilateral segmental PE, three patients had bilateral segmental PE, one patient had main pulmonary artery trunk PE and one patient had a saddle PE (Figure 1). Four patients were clinically asymptomatic and six were symptomatic with cardiorespiratory symptoms of PE.

We suspected VTE causing PE and paradoxical embolism via a patent foramen ovale in three patients based upon the quartet of concurrent findings: DVT, PE identified at stroke admission, patent foramen ovale demonstrated on echocardiography and

\section{Table 1: Baseline characteristics of patients}

\begin{tabular}{l|l|l}
\hline 1 & Age in years (median [range]) & $61(25-88)$ \\
\hline 2 & Female sex $(\%[n / N])$ & $50(5 / 10)$ \\
\hline 3 & Medical history $(\%[n / N])$ & $40(4 / 10)$ \\
\hline & HTN & $10(1 / 10)$ \\
\hline & DM & $10(1 / 10)$ \\
\hline & OCP & $30(3 / 10)$ \\
\hline & DLP & $40(4 / 10)$ \\
\hline 3 & Smoking & $14(4-24)$ \\
\hline 4 & NIHSS $($ median $[$ range]) & $30(3 / 10)$ \\
\hline 5 & ECHO-PFO $(\%[n / N])$ & $70(7 / 10)$ \\
\hline 6 & Doppler DVT $(\%[n / N])$ & $60(6 / 10)$ \\
\hline 7 & Symptomatic PE $(\%[n / N])$ & $30(3 / 10)$ \\
\hline & Outcomes $(\%[n / N])$ & $30(3 / 10)$ \\
\hline & Good & $40(4 / 10)$ \\
\hline & Poor & Death
\end{tabular}

$\mathrm{HTN}=$ hypertension $; \mathrm{DM}=$ diabetes mellitus OCP $=$ oral contraceptive pills; DLP $=$ dyslipidemia; NIHSS $=$ National Institutes of Health Stroke Scale range from 0 to 42 , with higher scores indicating more severe neurologic deficits; ECHO-PFO = echocardiogram positive for patent foramen ovale; $\mathrm{DVT}=$ deep vein thrombosis; $\mathrm{PE}=$ pulmonary embolism. embolic ischemic stroke. Clinically we suspected thrombophilia as an underlying mechanism in four patients but a complete thrombophilia work up was not done in all patients. All patients were treated with intravenous unfractionated heparin except one patient who developed thrombocytopenia and was switched to argatroban. Outcome at discharge was death in four, significant neurological disability in three (modified ranking scale score 4-5) and the remaining three patients had good and independent clinical recovery. The cause of death in two patients was massive $\mathrm{PE}$ and malignant middle cerebral artery (MCA) stroke with herniation in two others.

Pulmonary embolism was far less common in this modern series occurring at half the prevalence compared with prior studies. ${ }^{2}$ From the Registry of the Canadian Stroke Network, Pongmoragot et al. ${ }^{9}$ included all AIS patients admitted to participating institutions and among 11,287 patients with AIS, PE was found in $89(0.78 \%)$. Pulmonary embolism was more common among patients with severe stroke, history of cancer, previous DVT/PE. Pulmonary embolism was associated with lower long-term survival, greater disability and longer length of stay. Importantly most PE was detected concurrently at presentation rather than as a consequence of stroke-related hemiplegia, and both symptomatic and asymptomatic PE were identified using the stroke protocol CTA. Modern prevention using both stroke unit care with early mobilization, combined with enoxaparin or intermittent pneumatic compression stockings, is the likely reason for the reduced the rate of PE. Further, the acute treatment of stroke, both medically with thrombolysis and endovascularly, may have resulted in a reduction in the proportion of strokes with high-risk hemiplegia.

Most patients in our series had segmental or subsegmental PE. The clinical implications of these subsegmental PE, which are more likely to be asymptomatic, are unknown. In an analysis of the 383 pulmonary angiograms from the PIOPED study, which included patients with symptomatic presentations, the proportion of subsegmental PE was only $6 \%{ }^{10}$ The greater use of CTA imaging, means that segmental and subsegmental PE can be detected incidentally. Although all patients with a PE diagnosis were treated in our center, there is a lack of consensus on whether this is necessary. The risk of hemorrhage may be higher than the risk of major thromboembolic complications. ${ }^{11,12}$ In the setting of concurrent acute stroke, PE resulting in hypoxia can worsen existing cerebral ischemia. Medical thrombolysis (with alteplase using the stroke dosing protocol) may solve both problems, but the subsequent decision to anticoagulate or not requires careful judgment. Heparins are generally not used in stroke because they are associated with a greater risk of major hemorrhage ${ }^{13}$ and withholding heparins in the setting PE may expose the patient to a risk of a major thromboembolic event.

Limitations of our study include the standard CTA technique, which only includes the proximal pulmonary arteries and upper lobes. We may have missed lower lobe pulmonary emboli. Further, the small number of events (PE) identified warrants confirmation in future studies.

Pulmonary embolism is now increasingly rare in AIS. However, it may be a marker of an underlying pro-thrombotic state, can be discovered at presentation with modern imaging, be uncommonly associated with a right-to-left intracardiac or intrapulmonary shunt and portends a poorer prognosis. Further work is needed on therapeutic strategies. Stroke physicians, emergency physicians and radiologists should be aware of and look for PE on stroke protocol CTA studies, concurrently with AIS imaging evaluation. 


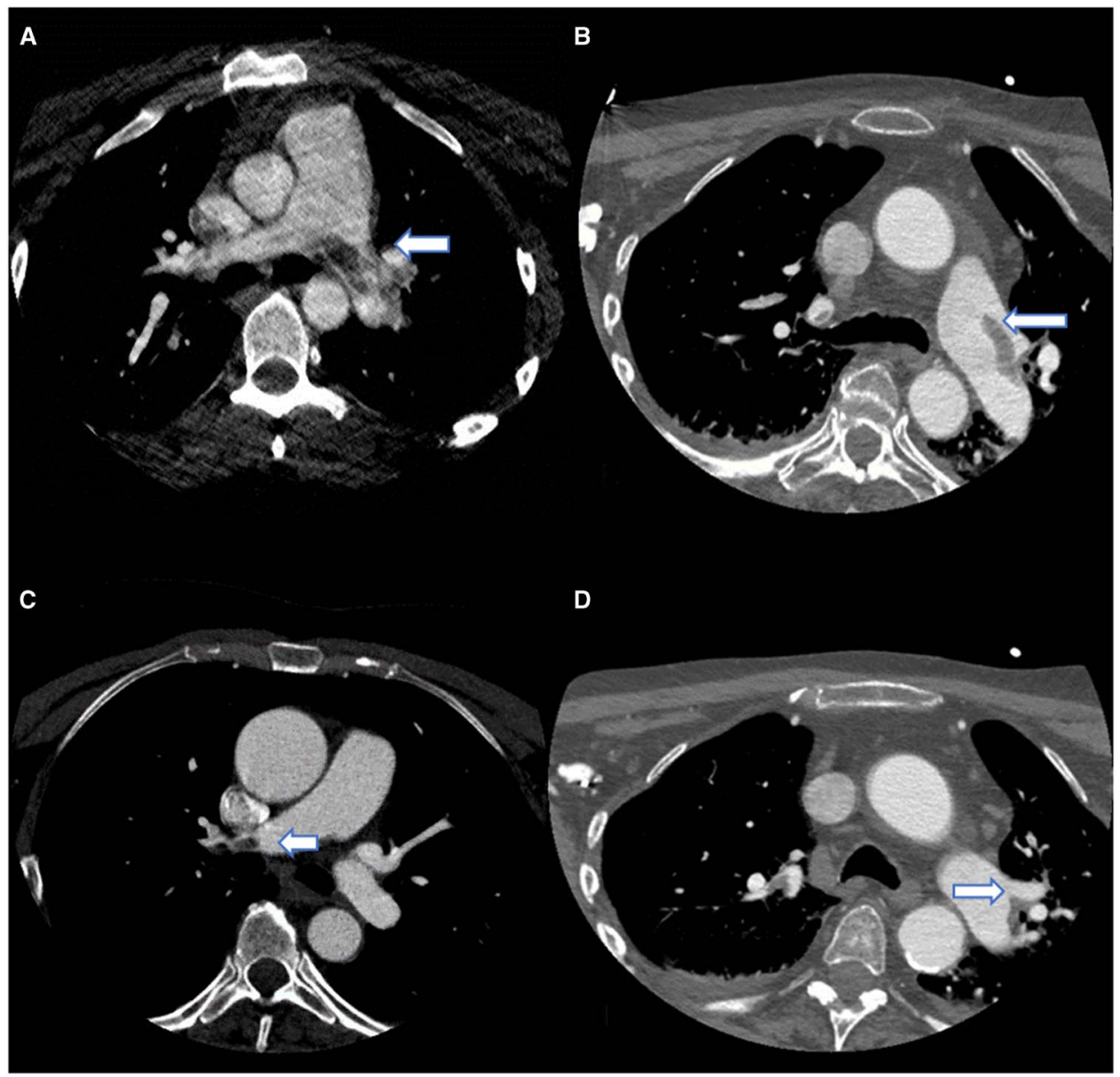

Figure 1: $C T$ angiogram and neck stroke protocol showing patients from this series with (A) saddle pulmonary embolus $[P E],(B)$ segmental $P E$, and $(C)$ subsegmental $P E$.

\section{Statement of AUthorship}

PVE and MDH reviewed concept and design, drafted the manuscript, and provided critical revision of the manuscript content. DS and SD drafted the manuscript and provided critical revision of the manuscript. All authors read and approved the final manuscript.

\section{Disclosures}

The authors have nothing to disclose.

\section{REFERENCES}

1. Naess IA, Christiansen SC, Romundstad P, Cannegieter SC, Rosendaal FR, Hammerstrøm J. Incidence and mortality of venous thrombosis: a populationbased study. J Thromb Haemost. 2007;5:692-9.

2. Skaf E, Stein P, Beemath A, Sanchez J, Bustamante M, Olson R. Venous thromboembolism in patients with ischemic and hemorrhagic stroke. Am J Cardiol. 2005;96(12):1731-3.

3. Carson JL, Kelley MA, Duff A, et al. The clinical course of pulmonary embolism. N Engl J Med. 1992;326:1240-5.

4. Skaf E, Stein P, Beemath A, Sanchez J, Olson R. Fatal pulmonary embolism and stroke. Am J Cardiol. 2006;97(12):1776-7.

5. Davenport RJ, Dennis MS, Wellwood I, Warlow CP. Complications after acute stroke. Stroke. 1996;27:415-20.

6. Muir K. The PREVAIL trial and low-molecular-weight heparin for prevention of venous thromboembolism. Stroke. 2008;39(7): 2174-2176.
7. Dennis M, Sandercock P, Graham C, Forbes J. The Clots in Legs Or sTockings after Stroke (CLOTS) 3 trial: a randomised controlled trial to determine whether or not intermittent pneumatic compression reduces the risk of post-stroke deep vein thrombosis and to estimate its cost-effectiveness. Health Technol Assess. 2015; 19(76):1-90.

8. Hart RG, Diener H-C, Coutts SB, et al. Embolic strokes of undetermined source: the case for a new clinical construct. Lancet Neurol. 2014;13(4):429-38.

9. Pongmoragot J, Rabinstein A, Nilanont Y, Swartz R, Zhou L, Saposnik G. Pulmonary embolism in ischemic stroke: clinical presentation, risk factors, and outcome. J Am Heart Assoc. 2013; 2(6): $\mathrm{e} 000372$.

10. Stein PD, Henry JW. Prevalence of acute pulmonary embolism in central and subsegmental pulmonary arteries and relation to probability interpretation of ventilation/perfusion lung scans. Chest. 1997;111:1246-8.

11. Perrier A, Howarth N, Didier D, et al. Performance of helical computed tomography in unselected outpatients with suspected pulmonary embolism. Ann Intern Med. 2001;135:88-97.

12. Stein PD, Goodman LR, Hull RD, Dalen JE, Matta F. Diagnosis and management of isolated subsegmental pulmonary embolism: review and assessment of the options. Clin Appl Thromb Hemost. 2012;18:20-6.

13. Sandercock PAG, Counsell C, Kane EJ. Anticoagulants for acute ischaemic stroke. Cochrane Database Syst Rev. 2015;(3): CD000024. 